\title{
Bevacizumab Related Hypertension and Reversible Posterior Leukoencephalopathy Syndrome in Gynecologic Malignancies: A Case Control Study
}

\author{
Sarah N. Cross", Elena S. Ratner, Dan A. Silasi, Alessandro D. Santin, Masoud Azodi, \\ Thomas J. Rutherford, Peter E. Schwartz \\ Department of Obstetrics, Gynecology and Reproductive Sciences, Yale University School of Medicine, New Haven, USA. \\ Email: sarah.cross@yale.edu
}

Received March 20 ${ }^{\text {th }}, 2011$; revised April 21 ${ }^{\text {st }}, 2011$; accepted April 30 $0^{\text {th }}, 2011$.

\begin{abstract}
Background: Bevacizumab is increasingly being used in the treatment of gynecologic malignancies, but has significant side-effects including hypertension and reversible posterior leukoencephalopathy (RPLS), which must be recognized by the gynecologic oncologist. Methods: A 26-month institutional retrospective review of bevacizumab in the treatment of gynecologic malignancies. Patients were grouped according to whether they had bevacizumab-related hypertension (defined as at least a grade one hypertensive toxicity) or not. There were no differences in patient demographics between the groups. Risk factors for developing bevacizumab-related hypertension were assessed using t-tests, Wilcoxon rank sum test and Fisher's exact test. Results: Our group has treated 45 patients with bevacizumab. Fifteen (33\%) patients had a pre-existing diagnosis of hypertension, 12 (80\%) of whom had at least one elevated blood pressure during treatment. The $30(67 \%)$ patients who did not have a pre-existing diagnosis of hypertension still had a high incidence of bevacizumab-related elevated blood pressure $(14,47 \%)$. The majority of patients $(26,58 \%)$ had at least one therapy cycle complicated by hypertension. Patients who experienced bevacizumab-related hypertension were significantly more likely than not to have a history of hypertension (odds ratio of 4.6, 95\% CI 1.1-19.6). There was a 4.4\% incidence of reversible posterior leukoencephalopathy. Patients with age equal to or greater than 75 years, stage IV disease, and creatinine elevations greater than or equal to $1.4 \mathrm{mg} / \mathrm{dL}$ were significantly more likely to develop bevacizumab-related hypertension. Other factors such as numbers of prior chemotherapies, cycles of bevacizumab, BMI, cancer site, and histology were not significantly associated with bevacizumab-related hypertension. Conclusions: Hypertension is a problem for patients on bevacizumab whether or not they have a pre-existing diagnosis. However, those with a history of hypertension were significantly more likely to have bevacizumab-related hypertension.
\end{abstract}

Keywords: Ovarian Cancer, Bevacizumab, Hypertension, Posterior Reversible Leukoencephalopathy

\section{Introduction}

Ovarian cancer ranks fifth in cancer deaths among American women, accounting for more deaths than any other cancer arising in the female reproductive system. It is estimated that there will be 21,880 new cases and 13,850 deaths from ovarian cancer in the United States during 2010 [1]. Despite advances in surgical techniques and chemotherapy the overwhelming majority of women diagnosed to have advanced stage ovarian cancer will ultimately develop recurrent disease refractory to therapy and succumb to the disease. Since the introduction of paclitaxel in the 1980s [2-4] there have not been substantial therapeutic advances to the treatment of this disease.
Bevacizumab (Avastin; Genetech Inc, South San Francisco, CA) is a monoclonal humanized recombinant IgG1 antibody that binds and inhibits vascular endothelial growth factor (VEGF) [5]. Bevacizumab has been demonstrated to reduce tumor perfusion by inhibiting endothelial cell proliferation and angiogenesis [6]. Bevacizumab has FDA approval for use in metastatic colorectal cancer. Clinical trials are underway to evaluate its therapeutic efficacy in other solid tumors including renal cell carcinoma, metastatic breast cancer, metastatic non-small cell lung cancer, pancreatic and prostate cancer $[7,8]$, in addition to advanced ovarian cancer and primary peritoneal carcinoma.

One of the most common side-effects of bevacizumab 
is hypertension, which has been reported to occur in up to 67 percent of patients and can occur at any point during treatment [7]. Importantly the hypertension is dose dependant and is more commonly seen at higher doses (10 or $15 \mathrm{mg} / \mathrm{kg}$ ) versus lower doses $(3,5$ or $7.5 \mathrm{mg} / \mathrm{kg}$ ) of bevacizumab $[7,8]$. The hypertension associated with bevacizumab is usually easily managed [9] but is occasionally dose-limiting [10] with an incidence of grade 3 or 4 hypertension in 3 to 14.8 percent in ovarian cancer studies [11] (Table 1). Importantly, in addition to causing new hypertension, bevacizumab can worsen pre-existing hypertension [12]. Indeed, the hypertension associated with bevacizumab is so common that some have suggested using blood pressure as a clinical indicator for dose titration and drug efficacy $[7,10]$.

Reversible posterior leukoencephalopathy syndrome (RPLS) is clinically characterized by headache, nausea, vomiting, vision disturbances, cortical blindness, altered mental status and seizures. Patients often present with hypertension, may have renal disease, eclampsia, or be on immune-modulating or cytotoxic medications for malignancy or auto-immune conditions [13], such as cyclosporine [14]. Radiographically RPLS appears as posterior white matter lesions bilaterally in the parietotemporal-occipital region of the cerebral hemispheres without evidence of infarction or hemorrhage on brain imaging.

To date there have been several published case reports of RPLS in association with treatment with bevacizumab for malignancies including renal carcinoma [15], colorectal cancer [16-20], breast cancer [21], and pediatric hepatoblastoma [22]. A recent review of the literature estimates an incidence of RPLS of less than 0.1 percent in clinical approval studies [7].

The objective of this study was to investigate which factors increased a patient's likelihood for hypertension while undergoing treatment with bevacizumab. Specifically, we postulated that women who had co-existing renal disease, increased age, advanced stage disease, and extended treatment with bevacizumab (more than 6 cycles) would have a higher rate of bevacizumab-related hypertension. We also investigated the relationship be- tween numbers of prior chemotherapies, BMI, cancer site, histology and development of bevacizumab-related hypertension.

\section{Materials \& Methods}

This study is a twenty-six month retrospective chart review of the forty-five consecutive patients at the Yale Gynecologic Oncology Center treated with bevacizumab. Forty-two were on study; either Ovarian Cancer Evaluation of Avastin and Safety (OCEANS) or Gynecologic Oncology Group-218 (GOG-218) while three were offstudy. All patients were treated at a standard dose of 15 $\mathrm{mg} / \mathrm{kg}$ of actual weight, for ovarian, fallopian tube and primary peritoneal carcinoma either alone or in combination with other anti-cancer agents (bevacizumab plus either carboplatin and gemcitabine or carboplatin and paclitaxel). Patients were grouped according to whether they had bevacizumab-related hypertension (defined as at least a grade one hypertensive toxicity using the National Cancer Institute (NCI) common toxicity criteria for hypertension) (26 patients) or not (19 patients) (Table 1). There were no differences in patient demographics between the two groups as presented in Table 2. Risk factors for developing bevacizumab-related hypertension were assessed using t-tests, Wilcoxon rank sum test and Fisher's exact test.

\section{Results}

Twelve ( $80 \%$ ) of the 15 patients with a history of hypertension had bevacizumab-related hypertension during treatment $(>140 \mathrm{~mm} \mathrm{Hg}$ systolic and/or $>90 \mathrm{~mm} \mathrm{Hg}$ diastolic), including one in whom the hypertension was attributed to a paclitaxel reaction (Table 3). The $30 \mathrm{pa}-$ tients who did not have a pre-existing diagnosis of hypertension still had a high incidence of bevacizumab-related hypertension (14, 47\%) (Tables 3 and 4). In total, 26 patients $(58 \%)$ had bevacizumab-related hypertension (Table 3). Sixteen of the 19 (84\%) patients who did not experience bevacizumab-related hypertension lacked a history of hypertension (Table 4). Patients who experienced bevacizumab-related hypertension were more likely than not to have a history of hypertension

Table 1. National cancer institute common toxicity criteria [34].

\begin{tabular}{|c|c|c|c|c|c|}
\hline Criteria & 0 & 1 & 2 & 3 & 4 \\
\hline Description & None & $\begin{array}{l}\text { Asymptomatic, transient } \\
\text { increase by }>20 \mathrm{~mm} \mathrm{Hg} \\
\text { (diastolic) or to } \\
>150 / 100 \text { if previously } \\
\text { WNL; not requiring } \\
\text { treatment }\end{array}$ & $\begin{array}{l}\text { Recurrent or persistent } \\
\text { or symptomatic increase } \\
\text { by }>20 \mathrm{~mm} \mathrm{Hg} \\
\text { (diastolic) or to } \\
>150 / 100 \text { if previously } \\
\text { WNL; not requiring } \\
\text { treatment }\end{array}$ & $\begin{array}{l}\text { Requiring therapy or } \\
\text { more intensive therapy } \\
\text { than previously }\end{array}$ & Hypertensive crisis \\
\hline
\end{tabular}


Table 2. Patient demographics and characteristics.

\begin{tabular}{|c|c|c|c|c|}
\hline \multicolumn{2}{|l|}{ Demographic } & Bevacizumab-related HTN & No bevacizumab-related HTN & $P$-value \\
\hline \multicolumn{2}{|l|}{ Age (mean in years) } & $64.12 \pm 11.91$ & $58.47 \pm 8.89$ & $0.089^{*}$ \\
\hline \multicolumn{2}{|c|}{ Number of prior chemotherapies (median) } & $1(0-4)$ & $0(0-2)$ & $0.180^{\circ}$ \\
\hline \multicolumn{2}{|c|}{ Cycles of bevacizumab (median) } & $7(1-18)$ & $9(2-28)$ & $0.301^{\circ}$ \\
\hline \multicolumn{2}{|l|}{ BMI (mean, in $\mathrm{kg} / \mathrm{m}^{2}$ ) } & $29.83 \pm 6.34$ & $27.27 \pm 5.75$ & $0.172 *$ \\
\hline \multicolumn{2}{|c|}{ Baseline SBP (mean, in mm Hg) } & $136 \pm 18$ & $129 \pm 17$ & $0.190 *$ \\
\hline \multicolumn{2}{|c|}{ Baseline DBP (mean, in mm Hg) } & $78 \pm 11$ & $74 \pm 8$ & $0.131 *$ \\
\hline \multicolumn{2}{|l|}{ Baseline $\mathrm{Cr}$ (mean, in $\mathrm{mg} / \mathrm{dL}$ ) } & $0.89 \pm 0.41$ & $0.79 \pm 0.14$ & $0.314 *$ \\
\hline \multirow{2}{*}{ Cancer Site (\# of patients) } & Ovary & 22 & 16 & \multirow{2}{*}{$0.6822^{\circ}$} \\
\hline & Tubal \& Peritoneal & 3 & 4 & \\
\hline \multirow{2}{*}{ FIGO Stage (\# of patients) } & IIC, IIIA, IIIB & 3 & 4 & \multirow{2}{*}{$0.4329^{\circ}$} \\
\hline & IIIC, IV & 23 & 15 & \\
\hline \multirow{3}{*}{ Histology (\# of patients) } & Serous & 17 & 14 & \multirow{3}{*}{$0.7460^{\circ}$} \\
\hline & Mixed, Transitional, & & & \\
\hline & $\begin{array}{l}\text { Endometrioid, Clear Cell, } \\
\text { Undifferentiated }\end{array}$ & 9 & 5 & \\
\hline \multirow[b]{2}{*}{ Ethnicity (\# of patients) } & Caucasian & 24 & 17 & \multirow[b]{2}{*}{$0.841^{\bullet}$} \\
\hline & Hispanic, Indian, African & 2 & 2 & \\
\hline \multirow{2}{*}{ Comorbidity $^{+}$(\# of patients) } & Yes & 5 & 4 & \multirow{2}{*}{$0.821^{\bullet}$} \\
\hline & No & 21 & 15 & \\
\hline
\end{tabular}

*t-test; ${ }^{\circ}$ Wilcoxon rank sum; ${ }^{\bullet}$ Fisher's exact test; ${ }^{+}$diabetes mellitus or cardiovascular disease such as hypercholesterolemia or CAD.

Table 3. Incidence of elevated blood pressure (BP)* by history of hypertension.

\begin{tabular}{lllc}
\hline $\begin{array}{l}\text { History of } \\
\text { hypertension }\end{array}$ & No elevated BP & $\geq 1$ elevated BP & Total \\
\hline None (30) & $16(53 \%)$ & $14(47 \%)$ & $30(100 \%)$ \\
Positive history $(15)$ & $3(20 \%)$ & $12(80 \%)$ & $15(100 \%)$ \\
Total (45) & $19(42 \%)$ & $26(58 \%)$ & $45(100 \%)$ \\
\hline
\end{tabular}

*At least one blood pressure $>140 \mathrm{~mm} \mathrm{Hg}$ systolic and/or $>90 \mathrm{~mm} \mathrm{Hg}$ diastolic.

Table 4. Prevalence of hypertension in patients with and without elevated blood pressure (BP)*.

\begin{tabular}{llll}
\hline Blood Pressure & $\begin{array}{l}\text { No history of } \\
\text { hypertension }\end{array}$ & $\begin{array}{l}\text { History of } \\
\text { hypertension }\end{array}$ & Total \\
\hline No elevated BP (19) & $16(84 \%)$ & $3(16 \%)$ & $19(100 \%)$ \\
$\geq 1$ elevated BP (26) & $14(54 \%)$ & $12(46 \%)$ & $26(100 \%)$ \\
Total (45) & $30(67 \%)$ & $15(33 \%)$ & $45(100 \%)$ \\
Odds & 0.875 & 4 & $4.6(95 \% \mathrm{CI}=$ \\
& & $4.1-19.6)$ \\
\hline
\end{tabular}

*At least one blood pressure $>140 \mathrm{~mm} \mathrm{Hg}$ systolic and/or $>90 \mathrm{~mm} \mathrm{Hg}$ diastolic.

(12, $46 \%$ versus $14,54 \%$ ) with a significant odds ratio of $4.6(95 \% \mathrm{CI}=1.1-19.6 ; p$-value $=0.05)($ Tables 4 and 5$)$.

Sixteen patients had grade 1 elevations in blood pressure not requiring initiation of medication or postponement of treatment, including one in whom the hypertension was attributed to a paclitaxel reaction, one who later had a bowel perforation and one who had a seizure while undergoing treatment and was diagnosed with new brain metastases (Table 1). Three patients required at least one cycle to be held in the setting of grade 2 hypertension, all of whom had pre-existing diagnoses and were on antihypertensive medications. Four additional patients had grade 3 hypertension and were initiated on anti-hypertensive medications during or after treatment, none of whom had a pre-existing diagnosis, including one who subsequently required a cycle to be held due to hypertension. One patient had treatment complicated by RPLS and one by hypertension requiring discontinuation of bevacizumab. Lastly, one patient had bevacizumab discontinued due to hypertension and presented 8 weeks later with an encephalopathy, an intracranial bleed and a fatal bowel perforation. In this small series the incidence of reversible posterior leukoencephalopathy was $4.4 \%$.

Patients with age equal to or greater than 75 years were significantly more likely to have hypertensive toxicity while on bevacizumab with a $p$-value of 0.03 (Table 5). Patients with stage IV disease were also significantly more likely to have bevacizumab-related hypertension with an odds ratio of $9(95 \% \mathrm{CI}=1.02-80$; $p$-value $=0.03)($ Table 5). Patients whose creatinine peaked at greater than or equal to $1.4 \mathrm{mg} / \mathrm{dL}$ were significantly more likely to have bevacizumab-related hypertension with a $p$-value of 0.03 (Table 5). Other factors such as numbers of prior chemotherapies, cycles of bavcizumab, BMI, cancer site, and histology were not significantly related with development of bevacizumabrelated hypertension (Table 5). 
Table 5. Results of patient demographics and bevacizumab-related hypertension.

\begin{tabular}{|c|c|c|c|c|}
\hline \multicolumn{2}{|l|}{ Demographic } & Bevacizumab-related HTN & No bevacizumab-related HTN & $P$-value \\
\hline \multicolumn{2}{|l|}{ Highest SBP (median, in mm Hg) } & $153(141-204)$ & $130(99-139)$ & $<0.005^{\circ}$ \\
\hline \multicolumn{2}{|l|}{ Highest DBP (median, in mm $\mathrm{Hg}$ ) } & $90(75-126)$ & $80(58-89)$ & $0.01^{\circ}$ \\
\hline \multicolumn{2}{|l|}{ Age $\geq 75$ (number of patients) } & 6 & 0 & $0.03^{\bullet}$ \\
\hline \multicolumn{2}{|l|}{$\mathrm{Cr} \geq 1.4 \mathrm{mg} / \mathrm{dL}$ (number of patients) } & 7 & 0 & $0.03^{\circ}$ \\
\hline \multicolumn{2}{|l|}{ Demographic } & Odds Ratio & $95 \%$ CI & $P$-value \\
\hline \multicolumn{2}{|l|}{ History of hypertension } & 4.6 & $1.07-19.5$ & $0.05^{\circ}$ \\
\hline \multicolumn{2}{|l|}{ Stage IV disease } & 9 & $1.02-80$ & $0.03^{\circ}$ \\
\hline Number of prior chemotherapies & $\geq 2$ vs $<2$ & 2.34 & $0.22-24$ & $0.63^{\circ}$ \\
\hline Cycles of bevacizumab & $\leq 6 \mathrm{vs}>6$ & 1.86 & $0.54-6.40$ & $0.37^{\bullet}$ \\
\hline BMI (in $\mathrm{kg} / \mathrm{m}^{2}$ ) & $\geq 30 \mathrm{vs}<30$ & 2.05 & $0.57-7.41$ & $0.35^{\circ}$ \\
\hline Cancer site & Ovary v other & 1.03 & $0.20-5.26$ & $1.0^{\circ}$ \\
\hline Histology & Serous $v$ other & 0.87 & $0.23-3.26$ & $1.0^{\circ}$ \\
\hline Ethnicity & Caucasian v other & 1.41 & $0.18-11.0$ & $1.0^{\circ}$ \\
\hline Comorbidity $^{+}$ & v none & 0.91 & $0.24-3.43$ & $1.0^{\circ}$ \\
\hline
\end{tabular}

${ }^{\circ}$ Wilcoxon rank sum; ${ }^{\circ}$ Fisher's exact test; ${ }^{+}$diabetes mellitus or cardiovascular disease such as hypercholesterolemia or CAD.

\section{Discussion}

As increasing numbers of patients with gynecologic malignancies are treated with bevacizumab, early recognition of the hypertension associated with it and the signs and symptoms of RPLS will be required by treating physicians. There is some evidence that this clinical syndrome is more prevalent in women [23,24] making awareness among gynecologic oncologists even more important. Guidelines for monitoring patients undergoing treatment with bevacizumab have been suggested including routine monitoring of blood pressure with initiation of antihypertensive medications as needed [25]. Specifically, patients who develop grade 1 hypertension can be continued on bevacizumab with weekly blood pressure monitoring [11]. Those with grade 2 hypertension need to be initiated on antihypertensives with bevacizumab held if they are symptomatic $[11,26]$. Those with grade 3 hypertension require escalation of their antihypertensives (dose increase or addition of a second agent) with bevaizumab held until they have asymptomatic grade 2 hypertension [11]. Lastly, those with grade 4 hypertension (including RPLS) require permanent discontinuation of bevacizumab [11,17].

Since bevacizumab inhibits VEGF, and therefore vasodilatation, the mechanism of bevacizumab-induced hypertension has been postulated to be via a decrease in nitric oxide production, which leads to vasoconstriction and decreased sodium excretion [7]. An alternative mechanism has been described, vascular rarefaction, which is a functional decrease in arterioles and capillaries that causes increased peripheral vascular resistance [7]. The latter process takes time to develop and resolve and therefore cannot account for the sometimes rapid clinical development and resolution of hypertension seen with bevacizumab [10]. Additionally, bevacizumab has been postulated to precipitate hypertension by inducing vasospasm [26].

These mechanisms may lead to a hypertensive crisis, in which severe blood pressure elevations are associated with endothelial failure, release of pro-inflammatory molecules, and dysregulation of the normal balance between endogenous vasodilatory mechanisms and vasoconstriction [27]. Treating bevacizumab-associated hypertension with vasodilating medications including angiotensin converting enzyme inhibitors (ACEIs) and calcium channel blockers (CCBs) has been widely described $[7,10]$, although it is unclear which class of agents is best.

The pathophysiology of RPLS, first described by Hinchey et al., is believed to be cerebral edema without infarction due to disruption of the vascular endothelium leading to a brain capillary leak syndrome [13]. Severe and sudden elevations in blood pressure which exceed the autoregulatory capability of the brain vasculature (usually at mean arterial blood pressures greater than 120 $\mathrm{mm} \mathrm{Hg}$ ) cause arteriolar vasodilatation and endothelial dysfunction, leakage of tight junctions with capillary leakage and break down of the blood-brain barrier thereby allowing transudation of fluid and vasogenic edema $[28,29]$. The posterior circulation has less sympathetic innervation than the anterior circulation, which is supplied by the carotid vasculature. Thus, the vertebrobasilar system is more vulnerable to loss of vasoconstrictive properties as blood pressure increases [14,30]. In addition to severe hypertension, immune-modulating medications may contribute to the pathophysiology of the syndrome by disrupting the vascular endothelium of the blood-brain barrier either indirectly or by a direct cytotoxic effect $[13,28]$.

The treatment of this condition involves aggressive control of the blood pressure, anticonvulsants and elimi- 
nation or dose-reduction of immunosuppressive or cytotoxic therapy [28]. Blood pressure reduction should follow the same guidelines as those in hypertensive encephalopathy (reduction by 20 to 25 percent within the first one to two hours or diastolic blood pressure reduction to less than $100 \mathrm{~mm} \mathrm{Hg}$ ). Anticonvulsants are usually not required once radiographic evidence of RPLS is no longer present [28].

Importantly, despite some cases in the literature to the contrary [28] (including reported fatalities [31,32] sometimes in association with intracranial hemorrhage $[16,33])$, RPLS is reversible with patients showing near or complete resolution on follow-up neuroimaging as well as resolution of symptoms as soon as one week after presentation [13]. Prompt recognition is paramount as it is believed that its reversibility is related to immediate treatment and that delays in normalization of blood pressure or continued use of implicated medications may lead to permanent sequelae.

The specific mechanism for bevacizumab-related RPLS has yet to be elucidated, but has been postulated to work indirectly via a combination of hypertension and vasospasm versus direct cytotoxic effects on systemic and cerebral endothelium [10]. All but one case of bevacizumab-related RPLS reported rapid resolution of the syndrome with aggressive blood pressure control. The exception was a case where the patient was normotensive, but was also being treated with three additional chemotherapies [18]. Given the resolution in bevacizumab-related RPLS with normalization of blood pressure, despite a half-life of bevacizumab of 20 days, it seems reasonable to conclude that the syndrome is caused by the side-effect of hypertension and not a direct cytotoxic effect of the medication on the endothelium.

The reversibility of the syndrome with prompt recognition and control of blood pressure and the frequency of bevacizumab-induced hypertension with increasing reports of RPLS associated with bevacizumab mandate fastidious attention to patient's blood pressure as they are undergoing treatment. The treating physicians should have a low threshold to initiate antihypertensives and, if that therapy fails to control blood pressure, to dose-reduce or terminate bevacizumab therapy in patients who have escalating hypertension. We initiated patients on antihypertensives if they had blood pressure elevations to $>$ $140 \mathrm{~mm} \mathrm{Hg}$ systolic or $>90 \mathrm{~mm} \mathrm{Hg}$ diastolic on more than one occasion, usually starting with an ACEI.

\section{Conclusions}

It is important to note that hypertension is a problem for patients on bevacizumab whether or not they have a preexisting diagnosis of hypertension. It can arise de-novo during treatment or worsen pre-existing hypertension. In our experience, patients with or without preexisting hypertension had a high risk of elevated blood pressure during bevacizumab treatment, but those with a pre-existing diagnosis were significantly more likely to have hypertensive complications. This small study suggests that clinicians should be even more vigilant in monitoring patients older than 75 years, those with stage IV disease, and those with creatinine toxicity (equal to or greater than $1.4 \mathrm{mg} / \mathrm{dL}$ ) while on bevacizumab.

Despite its small size this study demonstrates that hypertension can be a real problem for patients undergoing treatment with bevaziumab. Larger scale prospective studies will be needed to better characterize the nature of the risk factors associated with bevaizumab-related hypertension. Further studies will also be needed to investigate the relationship between development of hypertension and RPLS.

\section{Competing Interests}

The authors declare that they have no competing interests.

\section{Acknowledgements}

There were no sources of funding for this study.

\section{REFERENCES}

[1] A. Jemal, R. Siegel, J. Xu and E. Ward, "Cancer Statistics, 2010,” CA: A Cancer Journal for Clinicians, Vol. 60, No. 5, September-October 2010, pp. 277-300. doi:10.3322/caac. 20073

[2] J. McLaughlin, R. Miller, R. Powell and C. Smith, "19-Hydroxybaccatin III, 10-Deacetylcephalomannine, and 10-Deacetyltaxol: New Antitumor Taxanes from Taxus Wallichiana," Journal of Natural Products, Vol. 44, No. 3, May-June 1981, pp. 312-319. doi: $10.1021 / \mathrm{np} 50015 \mathrm{a} 013$

[3] N. Corvaja, A. Di Luzio, S. Biocca, A. Cattaneo and P. Calissano, "Morphological and Ultrastructural Changes in PC12 Pheochromocytoma Cells Induced by a Combined Treatment with NGF and Taxol," Experimental Cell Research, Vol. 142, No. 2, December 1982, pp. 385-395. doi:10.1016/0014-4827(82)90380-9

[4] P. Wiernik, E. Schwartz, J. Strauman, J. Dutcher, R. Lipton and E. Paietta, "Phase I Clinical and Pharmacokinetic Study of Taxol," Cancer Research, Vol. 47, No. 9, May 1987, pp. 2486-2493.

[5] Y. Wang, D. Fei, M. Vanderlaan and A. Song, "Biological Activity of Bevacizumab, a Humanized Anti-VEGF Antibody in vitro," Angiogenesis, Vol. 7, No. 4, May 2004, pp. 335-345. doi:10.1007/s10456-004-8272-2

[6] H. He, V. Venema, X. Gu, R. Venema, M. Marrero and R. Caldwell, "Vascular Endothelial Growth Factor Signals Endothelial Cell Production of Nitric Oxide and Prostacy- 
clin Through flk-1/KDR Activation of C-Src," Journal of Biological Chemistry, Vol. 274, No. 35, August 1999, pp. 25130-25135. doi:10.1074/jbc.274.35.25130

[7] S. Gressett and S. Shah, "Intricacies of Bevacizumab-Induced Toxicities and Their Management," Ann Pharmacother, Vol. 43, No. 3, March 2009, pp. 490-501. doi:10.1345/aph.1L426

[8] X. Zhu, S. Wu, W. Dahut and C. Parikh, "Risks of Proteinuria and Hypertension with Bevacizumab, an Antibody against Vascular Endothelial Growth Factor: Systematic Review and Meta-Analysis," American Journal of Kidney Diseases, Vol. 49, No. 2, February 2007, pp. 186-193. doi:10.1053/j.ajkd.2006.11.039

[9] F. Kabbinavar, J. Schulz, M. McCleod, T. Patel, J. T. Hamm, J. R. Hecht, R. Mass, B. Perrou, B. Nelson and W. F. Novotny, "Addition of Bevacizumab to Bolus Fluorouracil and Leucovorin in First-Line Metastatic Colorectal Cancer: Results of a Randomized Phase II Trial," Journal of Clinical Oncology, Vol. 23, No. 16, June 2005, pp. 3697-3705. doi:10.1200/JCO.2005.05.112

[10] F. Eskens and J. Verweij, "The Clinical Toxicity Profile of Vascular Endothelial Growth Factor (VEGF) and Vascular Endothelial Growth Factor Receptor (VEGFR) Targeting Angiogenesis Inhibitors a Review," European Journal of Cancer, Vol. 42, No. 18, December 2006, pp. 3127-3139. doi:10.1016/j.ejca.2006.09.015

[11] L. Randall and B. Monk, "Bevacizumab Toxicities and Their Management in Ovarian Cancer," Gynecologic Oncology, Vol. 117, No. 3, June 2010, pp. 497-504. doi:10.1016/j.ygyno.2010.02.021

[12] A. Pande, J. Lombardo, E. Spangenthal and M. Javle, "Hypertension Secondary to Anti-Angiogenic Therapy: Experience with Bevacizumab," Anticancer Research, Vol. 27, No. 5B, September-October 2007, pp. 34653470 .

[13] J. Hinchey, C. Chaves, B. Appignani, J. Breen, L. Pao, A. Wang, M. Pessin, C. Lamy, J. Mas and L. Caplan, "A Reversible Posterior Leukoencephalopathy Syndrome," New England Journal of Medicine, Vol. 334, No. 8, February 1996 , pp. 494-500. doi:10.1056/NEJM199602223340803

[14] J. Port and N. Beauchamp, "Reversible Intracerebral Pathologic Entities Mediated by Vascular Autoregulatory Dysfunction," Radiographics, Vol. 18, No. 2, MarchApril 1998, pp. 353-367.

[15] P. Glusker, L. Recht and B. Lane, "Reversible Posterior Leukoencephalopathy Syndrome and Bevacizumab," New England Journal of Medicine, Vol. 354, No. 9, March 2006, pp. 980-982. doi:10.1056/NEJMc052954

[16] R. Schwartz, S. Bravo, R. Klufas, L. Hsu, P. Barnes, C. Robson and J. Antin, "Cyclosporine Neurotoxicity and Its Relationship to Hypertensive Encephalopathy: CT and MR Findings in 16 Cases," American Journal of Roentgenology, Vol. 165, No. 3, September 1995, pp. 627-631.

[17] J. Allen, A. Adlakha and P. Bergethon, "Reversible Posterior Leukoencephalopathy Syndrome after Bevacizumab/FOLFIRI Regimen for Metastatic Colon Cancer,"
Archives of Neurology, Vol. 63, No. 10, October 2006, pp. 1475-1478. doi:10.1001/archneur.63.10.1475

[18] S. Peter, N. Hausmann, A. Schuster and H. Boehm, "Reversible Posterior Leukoencephalopathy Syndrome and Intravenous Bevacizumab," Clinical Experimental Ophthalmology," Vol. 36, No. 1, January-February 2008, pp. 94-96. doi:10.1111/j.1442-9071.2007.01658.x

[19] M. Koopman, E. Muller and C. J. A. Punt, "Reversible Posterior Leukoencephalopathy Syndrome Caused by Bevacizumab: Report of a Case," Diseases of the Colon \& Rectum, Vol. 51, No. 9, September 2008, pp. 14251426.doi:10.1007/s10350-008-9282-8

[20] G. El Maalouf, E. Mitry, A. Lacout, A. Livre and P. Rougier, "Isolated Brainstem Involvement in Posterior Reversible Leukoencephalopathy Induced by Bevacizumab," Journal of Neurology, Vol. 255, No. 2, February 2008, pp. 295-296. doi:10.1007/s00415-008-0692-2

[21] F. Burki, K. Badie, P. Bartoli, P. Bernard, J. Montastruc and H. Bagheri, "Reversible Posterior Leukoencephalopathy Syndrome Associated with Bevacizumab/Doxorubicin Regimen," British Journal of Clinical Pharmacology, Vol. 65, No. 5, May 2008, pp. 793-794. doi:10.1111/j.1365-2125.2008.03119.x

[22] C. Levy, K. Oo, F. Fireman, L. Pierre, M. Bania, S. Sadanandan, D. Yamashiro and J. L. G. Bender, "Reversible posterior Leukoencephalopathy Syndrome in a Child Treated with Bevacizumab," Pediatric Blood Cancer, Vol. 52, No. 5, May 2009, pp. 669-671. doi: $10.1002 / \mathrm{pbc} .21866$

[23] M. Marinella and R. Markert, "Reversible Posterior Leukoencephalopathy Syndrome Associated with Anticancer drugs," Internal Medicine Journal, Vol. 39, No. 12, December 2008, pp. 826-834. doi:10.1111/j.1445-5994.2008.01829.x

[24] H. Ay, F. Buonanno, P. Schaefer, D. Le, B. Wang, R. Gonzalez and W. Koroshetz, "Posterior Leukoencephalopathy without Severe Hypertension: Utility of Diffusion-Weighted MRI," Neurology, Vol. 51, No. 5, November 1998, pp. 1369-1376.

[25] S. Shord, L. Bressler, L. Tierney, S. Cuellar and A. George, "Understanding and Managing the Possible Adverse Effects Associated with Bevacizumab," American Journal of Health-System Pharmacy, Vol. 66, No. 11, June 2009, pp. 999-1013.

[26] C. Ozcan, S. Wong and P. Hari, "Reversible Posterior Leukoencephalopathy Syndrome and Bevacizumab," The New England Journal of Medicine, Vol. 354, No. 9, March 2006, pp. 980-982. doi:10.1056/NEJMc052954

[27] C. Vaughan and N. Delanty, "Hypertensive Emergencies," The Lancet, Vol. 356, No. 9227, July 2000, pp. 411-417. doi:10.1016/S0140-6736(00)02539-3

[28] V. Stott, M. Hurrell and T. Anderson, "Reversible Posterior Leukoencephalopathy Syndrome: A Misnomer Reviewed," Internal Medicine Journal, Vol. 35, No. 2, February 2005 , pp. 83-90. doi:10.1111/j.1445-5994.2004.00750.x 
[29] R. Garg, "Posterior Leukoencephalopathy Syndrome," Journal of Postgraduate Medicine, Vol. 77, No. 903, 2001, pp. 24-28. doi:10.1136/pmj.77.903.24

[30] R. Sheth, J. Riggs, J. Bodenstenier, A. Gutierrez, L. Ketonen and O. Ortiz, "Parietal Occipital Edema in Hypertensive Encephalopathy: A Pathogenic Mechanism," European Neurology, Vol. 36, No. 1, 1996, pp. 25-28. doi:10.1159/000117195

[31] M. Cain, G. Burton and R. Holcombe, "Fatal Leukoencephalopathy in a Patient with Non-Hodgkin's Lymphoma Treated with CHOP Chemotherapy and High-Dose Steroids," The American Journal of the Medical Sciences, Vol. 315, No. 3, March 1998, pp. 202-207. doi:10.1097/00000441-199803000-00011
[32] M. Greenwood, A. Dodds, R. Garricik and M. Rodriguez, "Posterior Leukoencephalopathy in Association with the Tumour Lysis Syndrome in Acute Lymphoblastic Leukaemia-A Case with Clinicopathological Correlation," Leukemia Lymphoma, Vol. 44, No. 4, April 2003, pp. 719-721. doi:10.1080/1042819031000060582

[33] R. Schwartz, “A Reversible Posterior Leukoencephalopathy Syndrome," The New England Journal of Medicine, Vol. 334, No 26, February 1996, p. 1743. doi:10.1056/NEJM199606273342613

[34] National Cancer Institute, "Common Toxicity Criteria," 1999. Internet Available: http://ctep.cancer.gov/protocol Development/electronic applications/docs/ctcv20 4-30-9 92.pdf. 